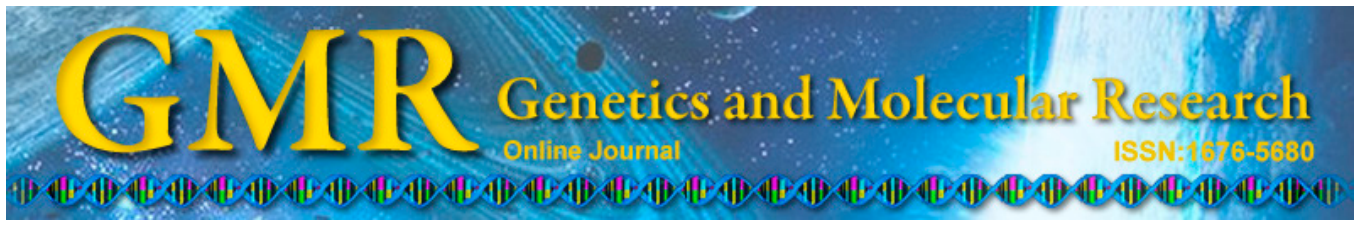

\title{
Hypolipidemic effect of safflower yellow and primary mechanism analysis
}

\author{
L.D. Bao ${ }^{1}$, Y. Wang ${ }^{1}$, X.H. Ren ${ }^{1}$, R.L. Ma ${ }^{1}$, H.J. Lv ${ }^{2}$ and B. Agula ${ }^{3}$ \\ ${ }^{1}$ Department of Pharmacy, \\ Affiliated Hospital of Inner Mongolia Medical University, Hohhot, China \\ ${ }^{2}$ Department of Scientific Research, \\ Affiliated Hospital of Inner Mongolia Medical University, Hohhot, China \\ ${ }^{3}$ College of Traditional Mongolia Medicine, \\ Inner Mongolia Medical University, Hohhot, China \\ Corresponding authors: H.J. Lv / B. Agula \\ E-mail: 1vhaijundsr@163.com / agula_b@163.com
}

Genet. Mol. Res. 14 (2): 6270-6278 (2015)

Received November 10, 2014

Accepted January 19, 2015

Published June 9, 2015

DOI http://dx.doi.org/10.4238/2015.June.9.14

\begin{abstract}
We examined the hypolipidemic effect of safflower yellow (SY) on hyperlipidemic mice and its influence on the biological synthesis of cholesterol in cells. Over 4 weeks, the levels of total cholesterol, triglyceride, low-density lipoprotein cholesterol, and highdensity lipoprotein cholesterol in serum were detected using a kit; mouse liver samples were acquired for paraffin sections, and mouse liver cells were observed under light microscope. Chinese hamster ovary cells were cultured in vitro, and an amphotericin B-cell model was adopted to observe the inhibitory effect of SY on the biological synthesis of intracellular cholesterol. An enzyme-linked immunosorbent assay was used to detect the survival rate of Chinese hamster ovary cells. The middle and high doses of SY significantly reduced the levels of total cholesterol, triglycerides, and low-density lipoprotein cholesterol in the serum of hyperlipidemic mice and low-density lipoprotein cholesterol/ high-density lipoprotein cholesterol ratio $(\mathrm{P}<0.05)$, and the fatty liver of hyperlipidemic mice was significantly alleviated. SY had a protective
\end{abstract}


effect on Chinese hamster ovary cells following amphotericin B injury $(\mathrm{P}<0.01)$. SY exerts significant hypolipidemic effects and prevents fatty liver in a mechanism associated with inhibition of the biosynthesis of intracellular cholesterol.

Key words: Cholesterol biosynthesis inhibition; Hypolipidemic effect; Fatty liver; Safflower yellow

\section{INTRODUCTION}

Atherosclerosis (AS) is the most common cardiovascular disease, which seriously affects human health. The morbidity and mortality rates of AS is increasing. Hyperlipidemia is an important cause of pathological changes in AS, and lipid metabolism disorders and abnormal lipoprotein composition are key factors in the pathogenesis of AS (Hyafil et al., 2012; Martinet et al., 2012; Zhao et al., 2012). Safflower yellow (SY), a natural yellow pigment extracted from safflower petals, is a chalcone compound. It is a natural, edible pigment approved in China and as a state-level new drug. Recently, Chinese and foreign scholars have conducted extensive and in-depth studies on the extraction and pharmacological effects of SY (Liu et al., 2011; Xi et al., 2012), and found that SY is not only a valuable natural edible pigment, with the advantages of attractive color, resistance to high temperatures, high pressure, low temperature, light, acid, and reduction, and antimicrobial properties, but also has many pharmacological properties such as expansion of coronary arteries, antioxidation, myocardial protection, lowering of blood pressure, immune suppression, and cerebral protection (Wang et al., 2011; Chen et al., 2012). In this study, we examined the effect of SY on the blood lipids of hyperlipidemic mice and the in vitro synthesis of cholesterol to provide a basis for its pharmacological applications.

\section{MATERIAL AND METHODS}

\section{Materials}

The materials included SY [Shanxi Huahui Kaide Pharmaceutical Co., Ltd. (Beijing, China), batch No. 20120725] and kits for determining total cholesterol (TC), triglyceride (TG), high-density lipoprotein cholesterol (HDL-C), and low-density lipoprotein cholesterol (LDL-C) (Wako Pure Chemical Industries, Ltd., Osaka, Japan). Simvastatin (SIM; Henan Dikang Pharmaceutical Co., Ltd., Henan, China) was prepared as a $15 \mathrm{mg} / \mathrm{mL}$ stock solution in dimethyl sulfoxide and stored at $-8^{\circ} \mathrm{C}$. Amphotericin B (North China Pharmaceutical Co., Ltd., Shanghai, China) was prepared as a $30 \mathrm{mg} / \mathrm{mL}$ stock solution in dimethyl sulfoxide, stored at $-8^{\circ} \mathrm{C}$, and used within 3 days. Lipoprotein-deficient serum was obtained from Biomedical Technologies, Inc., Stoughton, MA, USA. Mevalonic lactone was from Merck (White House Station, NJ, USA). The Chinese hamster ovary (CHO) cell line was from the Experimental Animal Center (Beijing University). The TC2323 $\mathrm{CO}_{2}$ incubator was from LEAD-TECH (Shanghai) Scientific Instrument Co., Ltd., Shanghai, China. The CH2-TKC-3 inverted optical microscope was from Darkoo Optics Co., Ltd., Guangdong, China. The DG-5031 enzyme-linked immunosorbent assay analyzer was from Midwest Taian Technology Service Co., Ltd. (Beijing, China). 


\section{Animal grouping and feeding}

Healthy adult male Kunming mice with a body mass of $25 \pm 2 \mathrm{~g}$ (provided by the Department of Laboratory Animal Science, Peking University) were selected and fed granular basal feed and tap water for 7 days. The body mass of the mice was determined, and the average body mass had increased to $28 \pm 2 \mathrm{~g}$. The mice were randomly divided into 6 groups with 15 mice in each group. There was no significant difference in the average body mass of the mice in each group according to analysis of variance $(\mathrm{P}>0.05)$. The groups were specifically divided as follows: normal control group (ND): fed with basal diet ( $20 \%$ soy protein, $45 \%$ corn flour, $30 \%$ wheat flour, $4 \%$ vegetable oil, $0.13 \%$ complex vitamins, $0.41 \%$ composite inorganic salt, and $0.46 \%$ salt); high-fat control group (HFD): fed with high-fat diet (15\% lard, $5 \%$ cholesterol, $0.5 \%$ cholate, and $79.5 \%$ basal feed); drug control group (SIM) + high-fat diet: simvastatin was administered at only $0.1 \mathrm{mg} /$ day per mouse orally; SY low-dose group: intragastric administration at $0.1 \mathrm{mg} /$ day per mouse; SY middle-dose group: intragastric administration at $0.5 \mathrm{mg} /$ day per mouse; SY high-dose group: intragastric administration at $1.0 \mathrm{mg} /$ day per mouse. The final 3 groups of mice were given a high-fat diet at the time of administration.

\section{Extraction of SY}

Because safflower yellow is freely soluble in water in weak acid conditions, the $\mathrm{pH}$ value of $50 \mathrm{~L}$ water was adjusted to weak alkaline conditions with potassium carbonate $\left(\mathrm{K}_{2} \mathrm{CO}_{3}\right)$, and then $\mathrm{SY}$ raw material was mixed into the water. SY was mixed with extracting solution for further extraction. The extract was placed into the settling tank for approximately $0.5 \mathrm{~h}$ to remove impurities at the bottom. The upper extract was removed and centrifuged, and the supernatant was dried by steaming on a water bath. The extraction process was generally repeated twice in order to improve SY yield. Because SY is unstable under alkaline conditions, the procedure was performed rapidly (Li et al., 2010; Monteiro et al., 2011).

\section{Blood lipid determination and pathological observation}

The feeding test lasted for 1 month, during which the mice were self-feeding and had free access to water. The mice were fasted for $12 \mathrm{~h}$ before the end of the test, and then blood was drawn by eye enucleation. Blood samples were collected, and serum was prepared. The levels of serum TC, TG, LDL-C, and HDL-C were detected using the kits. After blood sampling, the mice were euthanized immediately before removing the livers, which were dried with filter paper after washing with ice-cold normal saline; the livers were fixed in 5\% formalin, conventional paraffin-embedded, sectioned, and subjected to hematoxylin and eosin staining. The pathological changes in liver tissues were observed by light microscopy.

\section{Influence of SY on cholesterol biosynthesis in $\mathrm{CHO}$ cells}

$\mathrm{CHO}$ cells were suspended after digestion with $0.5 \%$ trypsin; the cell concentration was adjusted to $1 \times 10^{4}$ cells $/ \mathrm{mL}$ using liquid A (RPMI1640 medium containing $100 \mu \mathrm{g} / \mathrm{mL}$ glutamine and 5\% calf serum), and the cells were placed seeded on a 96-well plate with 200 $\mu \mathrm{L}$ in each well. At the same time, the normal control group (without amphotericin B), negative control group (equal volume of medium), positive control group (simvastatin at $1.0 \mathrm{mg}$ / 
$\mathrm{mL}$ ), and SY groups at different doses were established. Six replicate wells were used for each group. The cells were cultured in a $\mathrm{CO}_{2}$ incubator, and the medium was replaced with $100 \mu \mathrm{L}$ liquid B after cell adhesion (RPMI1640 medium containing 5\% lipoprotein-free serum and $100 \mu \mathrm{g} / \mathrm{mL}$ glutamine) for culturing for $48 \mathrm{~h}$, and then replaced with $100 \mu \mathrm{L}$ liquid C (liquid B was added $0.5 \mathrm{mM}$ mevalonate lactone, different concentrations of test samples, and the positive control drug). The cells were cultured for $48 \mathrm{~h}$, washed with phosphate-buffered saline, $\mathrm{pH} 7.2$, thrice, and $100 \mu \mathrm{L}$ liquid D was added (liquid B was added $100 \mu \mathrm{g} / \mathrm{mL}$ amphotericin

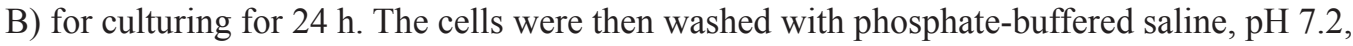
thrice. Next, $20 \mu \mathrm{L} 10 \mathrm{mg} / \mathrm{mL}$ MTT was added for continued cultivation for $8 \mathrm{~h}$ and then acidified. Fifty microliters of 5\% sodium dodecyl sulfate was added to terminate the reaction. The DG-5031-type enzyme-linked immunosorbent assay detector was used to detect the absorbance value (A value) at $570 \mathrm{~nm}$. A higher A value indicated higher survival of cells and stronger effects of the drug on the inhibition of cholesterol biosynthesis.

\section{Statistical analysis}

All data are reported as means \pm S.D and analyzed using the $t$ test. Data were processed using SPSS 12.5 (SPSS, Inc., Chicago, IL, USA).

\section{RESULTS}

\section{Influence of SY on body weight and food intake}

During the test, the average daily food intake of the mice in each group gradually increased, but no significant difference was observed among groups $(\mathrm{P}>0.05)$. The body mass of the mice showed an increase. On the 30th day, the body mass of the mice in the HFD group was higher than that in the other groups; the differences were significant in the SY high-dose and middle-dose groups $(\mathrm{P}<0.05)$. The body mass of all $\mathrm{SY}$ groups was lower than that of the ND group, but the difference was not significant $(P>0.05)$. With increasing dose, the rate of increase in body mass decreased, indicating that SY can inhibit the increase of body mass of mice fed HFD to varying degrees (Tables 1 and 2).

Table 1. Influence of safflower yellow (SY) on food intake (g/day).
\begin{tabular}{lccrrrr}
\hline Group & 5 th day & 10th day & 15th day & 20th day & 25th day & 30 th day \\
\hline ND & $7.7 \pm 1.9$ & $8.4 \pm 1.0$ & $9.5 \pm 0.9$ & $10.6 \pm 1.1$ & $11.1 \pm 1.3$ & $11.8 \pm 1.7$ \\
HFD & $7.5 \pm 1.3$ & $8.9 \pm 1.1$ & $9.8 \pm 1.5$ & $10.5 \pm 1.5$ & $11.3 \pm 1.0$ & $11.2 \pm 1.3$ \\
SIM & $7.2 \pm 1.2$ & $8.2 \pm 1.3$ & $10.1 \pm 1.7$ & $9.3 \pm 1.7$ & $11.2 \pm 1.6$ & $11.5 \pm 1.6$ \\
SY high-dose & $7.3 \pm 1.6$ & $8.5 \pm 1.7$ & $9.8 \pm 0.7$ & $10.9 \pm 1.3$ & $10.9 \pm 1.1$ & $11.1 \pm 1.5$ \\
SY middle-dose & $7.5 \pm 1.5$ & $8.9 \pm 1.2$ & $9.6 \pm 1.3$ & $9.8 \pm 1.2$ & $10.8 \pm 1.6$ & $11.6 \pm 1.7$ \\
SY low-dose & $7.6 \pm 1.7$ & $8.5 \pm 1.7$ & $10.0 \pm 1.4$ & $10.3 \pm 1.7$ & $11.3 \pm 1.5$ & $11.6 \pm 1.4$ \\
\hline
\end{tabular}

$\mathrm{ND}=$ normal control diet; HFD = high-fat diet; SIM = drug (simvastatin) group.

\section{Influence of SY on blood lipid levels}

The serum TG, TC, and LDL-C levels of mice in the HFD group were significantly higher than those of the ND group; a significant difference was observed $(\mathrm{P}<0.01)$, suggesting that the established animal model of hyperlipidemia was reliable. 
Table 2. Influence of safflower yellow (SY) on body weight (g).

\begin{tabular}{|c|c|c|c|c|c|c|}
\hline Group & 5th day & 10th day & 15 th day & 20th day & 25 th day & 30th day \\
\hline ND & $25.4 \pm 1.8$ & $26.3 \pm 1.7$ & $27.5 \pm 2.0$ & $28.6 \pm 1.7$ & $29.4 \pm 1.8$ & $29.8 \pm 1.6$ \\
\hline HFD & $25.1 \pm 1.4$ & $26.4 \pm 1.8$ & $27.4 \pm 1.9$ & $28.8 \pm 1.6$ & $30.1 \pm 1.9$ & $31.9 \pm 1.7$ \\
\hline SIM & $25.5 \pm 1.7$ & $25.9 \pm 1.8$ & $27.2 \pm 1.6$ & $28.7 \pm 1.8$ & $29.2 \pm 1.7$ & $29.7 \pm 1.9$ \\
\hline SY high-dose & $23.1 \pm 1.8$ & $24.4 \pm 1.7$ & $25.1 \pm 1.7$ & $25.9 \pm 1.4$ & $26.5 \pm 1.6$ & $27.2 \pm 1.4$ \\
\hline SY middle-dose & $22.9 \pm 1.9$ & $23.1 \pm 1.4$ & $23.4 \pm 1.5$ & $23.8 \pm 1.6$ & $24.0 \pm 1.4$ & $24.2 \pm 1.3^{*}$ \\
\hline SY low-dose & $23.6 \pm 1.7$ & $23.8 \pm 1.5$ & $24.0 \pm 1.6$ & $24.2 \pm 1.5$ & $24.3 \pm 1.6$ & $24.4 \pm 1.4^{*}$ \\
\hline
\end{tabular}

$* \mathrm{P}<0.05$ vs HFD group. For abbreviations, see Table 1.

Compared with the HFD group, serum TC and LDL-C levels of the mice in all SY groups were significantly reduced $(\mathrm{P}<0.05)$, and the differences were extremely significant in the middle-dose and high-dose groups $(\mathrm{P}<0.01)$. In the SY middle-dose and high-dose groups, serum TG levels were significantly decreased $(\mathrm{P}<0.05)$, while HDL-C level was significantly increased $(\mathrm{P}<0.05)$. In the $\mathrm{SY}$ low-dose group, TC and LDL-C levels were significantly decreased $(\mathrm{P}<0.05)$, but the difference in the TG and HDL-C levels was not significant $(\mathrm{P}>0.05)$. The levels of TC, TG, and LDL-C in all SY groups were higher than those in the SIM group, and the HDL-C level was higher only in the high-dose group than that in the SIM group. These results indicate that middle-dose and high-dose SY can significantly inhibit the increase in TC, TG, and LDL-C levels of mice in the high-fat model and significantly increase the HDL-C level.

The atherogenic index (AI) is the ratio of LDL-C and HDL-C. A lower AI value correlates to a decreased possibility of atherosclerosis incidence. The AI values of all SY groups were significantly lower than those of the HFD group $(\mathrm{P}<0.05)$, and only in the SY high-dose group, the AI value was lower than that in the ND group and higher than that in the SIM group (Table 3).

\begin{tabular}{|c|c|c|c|c|c|}
\hline Group & $\mathrm{TC}$ & TG & LDL-C & HDL-C & $\mathrm{AI}$ (LDL-C/HDL-C) \\
\hline ND & $2.49 \pm 0.84^{\# \#}$ & $0.97 \pm 0.31^{\# \#}$ & $0.81 \pm 0.21^{\# \#}$ & $1.15 \pm 0.21^{\# * *}$ & $0.82 \pm 0.25$ \\
\hline HFD & $5.04 \pm 1.12 * * \Delta \mathbf{\Delta}$ & $1.94 \pm 0.28 * * \mathbf{\Delta} \Delta$ & $1.42 \pm 0.17 * * \mathbf{\Delta} \mathbf{\Delta}$ & $1.92 \pm 0.29 * \mathbf{\Lambda}$ & $1.52 \pm 0.34 * * \mathbf{\Delta} \Delta$ \\
\hline SIM & $2.51 \pm 0.91^{\# \#}$ & $1.02 \pm 0.26^{\# \#}$ & $0.87 \pm 0.23^{\# \mathbf{\Lambda}}$ & $1.19 \pm 0.27^{\# \mathbf{\Delta} \Delta}$ & $0.78 \pm 0.23^{\# \#}$ \\
\hline SY high-dose & $4.14 \pm 1.05^{\# * \mathbf{\Delta}}$ & $1.81 \pm 0.11 * * \mathbf{\Lambda}$ & $1.26 \pm 0.29^{\# * *}$ & $1.64 \pm 0.24 * \Delta$ & $1.24 \pm 0.24^{* * * \Delta \mathbf{\Delta}}$ \\
\hline SY middle-dose & $3.61 \pm 0.81^{\# \boldsymbol{\Lambda}}$ & $1.44 \pm 0.23^{\# * \boldsymbol{\Lambda}}$ & $1.03 \pm 0.26^{\# *}$ & $1.32 \pm 0.29^{\# \mathbf{\Delta} \Delta}$ & $1.05 \pm 0.27^{\# *}$ \\
\hline SY low-dose & $2.47 \pm 0.81^{\# \#}$ & $1.13 \pm 0.14^{* * \boldsymbol{\Delta}}$ & $0.88 \pm 0.17^{\# \#}$ & $1.18 \pm 0.31^{\# \mathbf{\Delta} \Delta}$ & $0.80 \pm 0.19^{\# \#}$ \\
\hline
\end{tabular}

**P $<0.01, * \mathrm{P}<0.05$ vs SIM group; ${ }^{\wedge}{ }^{\star} \mathrm{P}<0.01,{ }^{\wedge} \mathrm{P}<0.05$ vs $\mathrm{ND}$ group; ${ }^{\#} \mathrm{P}<0.01,{ }^{\#} \mathrm{P}<0.05$ vs HFD group. For abbreviations, see Table 1 .

\section{Pathological changes in liver cells induced by SY}

In the ND group, hepatic tissue was essentially normal with a complete and clear structure. The structure of hepatic lobules was normal; hepatic cells were arranged regularly in the shape of hepatic cords and scattered in a radial pattern around the central vein. The structure of hepatic sinusoid was clear and showed no abnormal changes. Hepatic cells were polygon-shaped; the cytoplasm was uniform and rich, the cell membrane was clear, and the cell nucleus was large and centered, with a normal shape.

In the HFD group, the liver was enlarged. The surface color of tissues became yellow; and tissues spread densely in the shape of a dot. The liver had a round and blunt edge, similar 
to a nutmeg liver; the liver had a greasy texture. Hepatic lobules had an undefined dividing line; hepatic cords were arranged in a disordered fashion. Most hepatic sinusoids disappeared, most hepatic cells had severe steatosis, and the lobular peripheral part was the most severe (hepatic cells were swollen and became round; numerous lipid droplet vacuoles of different sizes and quantities could be observed in the cytoplasm). Cells containing fat vacuoles accounted for more than $2 / 3$ of the total number of hepatic cells. Under severe conditions, lipid droplets were integrated with each other, and the cell nucleus was squeezed to one side of the cells. The cell nucleus was squeezed to the side of cell membrane, as observed in adipose cell.

In the SY groups, the hepatic steatosis degree was decreased, while mice in the SY highdose and middle-dose groups had a light red liver. The liver of some mice was slightly enlarged, and no significant change could be observed just by observation. Clear structures of hepatic lobules and liver sinusoids and an orderly arrangement of cells could be observed under a low-power lens. Compared with the HFD group, lipid droplet vacuole hepatic cells containing fat vacuoles were reduced; small vacuoles could be observed around the nucleus in some cells, without cell fusion. The cell nucleus was in the middle of the cell, with an essentially normal shape and structure, which was approximately the same as normal hepatic cells. Regenerative hepatic cells could also be observed, and liver mesenchyme showed no fibrosis change. Compared with the model group, the hepatic steatosis degree of mice of all the administration groups was significantly improved and better than that of the positive control drug SIM group, as shown in Figure 1.
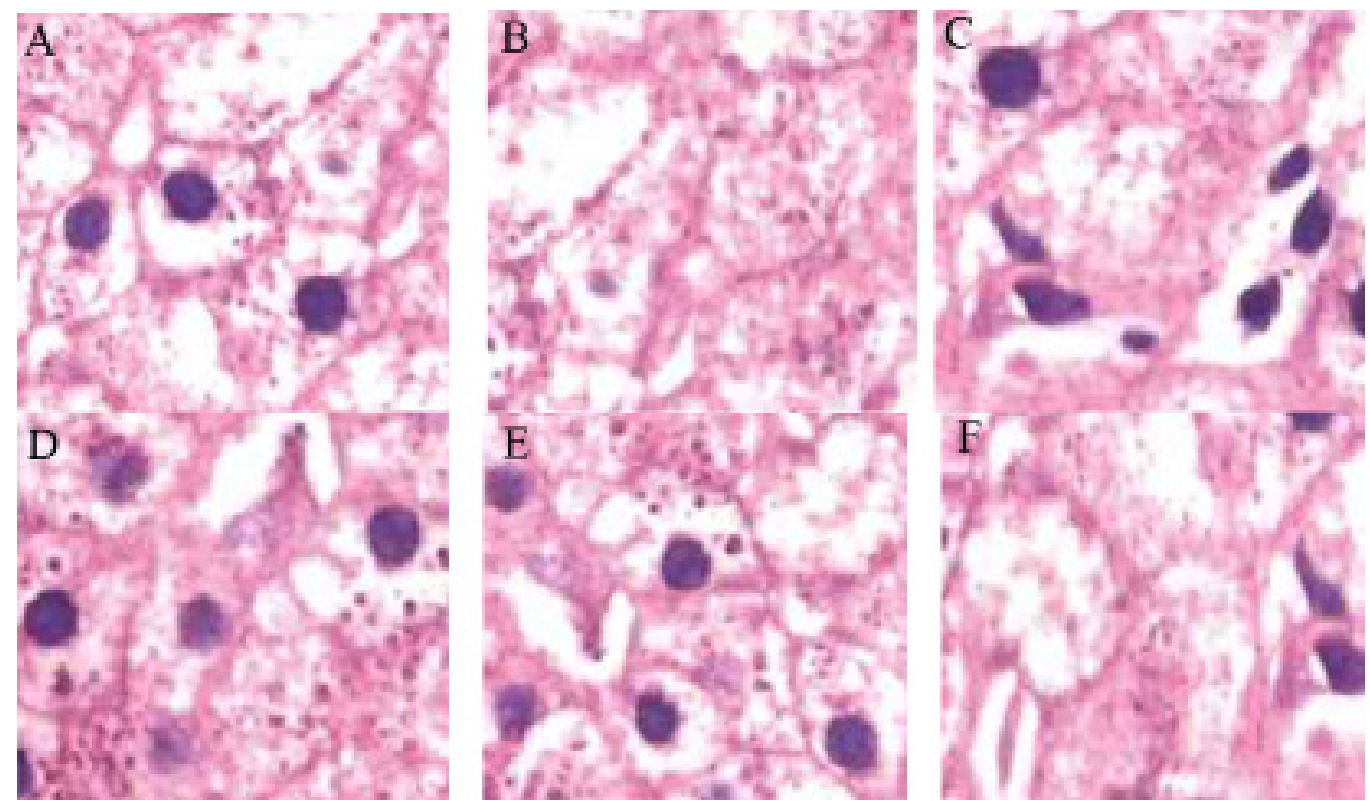

Figure 1. Hematoxylin and eosin staining results of liver tissues (200X). A. Normal control (ND) group; B. highfat control (HFD) group; C. Simvastatin (SIM) control group; D. Safflower yellow (SY) low-dose group; E. SY middle-dose group; F. SY high-dose group.

\section{Protective effects of SY on CHO cells against amphotericin B injuries}

The survival rate of CHO cells was detected using the MTT test. The effects of SY at 
the 3 dose levels on the survival rate of CHO cells are shown in Table 4. The results showed that compared with the normal control group, the difference between the SY high-dose and middle-dose groups was not significant $(\mathrm{P}>0.05)$, while the survival rate in the SY low-dose group was significantly lower than that in the normal control group, with the difference reaching an extremely significant level $(\mathrm{P}<0.01)$. Compared with the negative control group, the $\mathrm{A}_{570}$ of SY of the 3 levels were higher than that of the negative control group, and the difference was extremely significant $(\mathrm{P}<0.01)$; the $\mathrm{OD}_{570}$ value was the highest in the $\mathrm{SY}$ high-dose group, followed by the SY middle-dose group and the lowest in the SY low-dose group. There was no significant difference between the SY high-dose and middle-dose groups and the SIM group $(\mathrm{P}>0.05)$; while the difference was extremely significant between the SY low-dose group and SIM group $(\mathrm{P}<0.01)$, indicating that the $1 \mathrm{mg} / \mathrm{mL}$ SY high-dose and middle-dose groups protected $\mathrm{CHO}$ cells against the damage caused by amphotericin B. Although the $1 \mathrm{mg} /$ $\mathrm{mL} \mathrm{SY} \mathrm{low-dose} \mathrm{group} \mathrm{had} \mathrm{a} \mathrm{protective} \mathrm{effect} \mathrm{on} \mathrm{CHO}$ cells, the effect was lower than that of the high-dose and middle-dose groups at the same dose.

Table 4. Effects of safflower yellow (SY) on the survival rate of CHO cells.

\begin{tabular}{|c|c|c|}
\hline Group & Dose (mg/day) & Absorbance (A) \\
\hline ND & & $0.863 \pm 0.106^{\mathbf{\Delta}}$ \\
\hline Negative control & & $0.298 \pm 0.190^{\# *}$ \\
\hline SIM & 0.1 & $0.859 \pm 0.184^{\triangleleft}$ \\
\hline SY low-dose & 0.1 & $0.648 \pm 0.167^{\# \mathbf{\Delta} *}$ \\
\hline SY middle-dose & 0.5 & $0.779 \pm 0.179^{\mathbf{\Delta}}$ \\
\hline SY high-dose & 1.0 & $0.837 \pm 0.203^{\mathbf{\Delta}}$ \\
\hline
\end{tabular}

Compared with the normal control group, the optical density values of the low-dose and middle-dose groups in the SY middle-dose group and the low-dose group of the SY highdose group were lower, showing significant differences $(\mathrm{P}<0.05)$, and the difference between the other SY administration groups and the normal control group was not significant $(\mathrm{P}>$ 0.05). The value of each administration group was higher than that of the negative control group, and the differences reached an extremely significant level $(\mathrm{P}<0.01)$. The differences between the SY high-dose, middle-dose, and low-dose groups and the SIM group were statistically significant $(\mathrm{P}<0.05)$, and the differences between the other groups and SIM group were not significant $(\mathrm{P}>0.05)$.

\section{DISCUSSION}

Serum TC and TG levels are important indicators of hyperlipidemia. The main function of LDL is to transfer cholesterol synthesized in the liver to body tissues for use. The plasma LDL level, which showed a significant positive correlation with atherosclerosis, is thought to be a factor in inducing atherosclerosis (Angheloiu et al., 2012; Raz-Pasteur et al., 2012; Wei et al., 2012). The main function of HDL is to transfer cholesterol in the extrahepatic tissues back to the liver for metabolism. HDL can prevent cholesterol deposition in the arterial wall, is significantly negatively correlated with atherosclerosis incidence, and thus is considered an effective anti-atherosclerosis factor (Cacciapuoti, 2012; Kurihara et al., 2012). In the SY middle-dose and high-dose groups, the TC, LDL-C, and TG levels of hyperlipidemic mice were significantly reduced, while HDL-C level was increased. In the SY low-dose group, the 
TC and LDL-C levels were significantly decreased, but the TG and HDL-C levels showed no clear changes. A lower AI value indicates a lower atherosclerosis incidence. According to the test results, SY can significantly reduce the levels of serum TC, TG, LDL-C, and AI of highfat diet mice, significantly improving lipid metabolism, lowering blood lipids, and preventing atherosclerosis. Its lipid-lowering effect presents a clear dose-effect relationship. The results of pathological observation show that SY can reduce the fat pathological changes of liver cells to maintain normal hepatocyte morphology, affecting fatty liver.

The amphotericin B-cell model provides an effective and simple method for screening of endogenous cholesterol synthesis inhibitors. After complexes are formed by amphotericin $\mathrm{B}$ and cholesterol on the cell membrane, micropores appear, leading to cell membrane rupture and spillover of cytoplasmic contents (Balasegaram et al., 2012; Butsch et al., 2012). In the absence of supplied exogenous cholesterol, if endogenous cholesterol is used to synthesize inhibitors, the synthesis of endogenous cholesterol is inhibited, preventing the formation of the above complexes and protecting the cells against the damage caused by amphotericin B. In the amphotericin B-cell model, a higher A value indicates a larger number of live cells and less damage of the cells by amphotericin B. This indicates that in the absence of exogenous lipid, the synthesis of cell endogenous cholesterol is inhibited (Jung et al., 2012; Mohamed-Ahmed et al., 2012). The test results showed that the SY low-dose, middle-dose, and high-dose groups protected CHO cells from amphotericin B damage, in which a dose-effect relationship exists in the SY middle-dose and high-dose groups. This suggests that SY can significantly inhibit endogenous cholesterol synthesis, which may be one of the mechanisms regulating blood lipids.

\section{ACKNOWLEDGMENTS}

Research supported by the Nature Science Foundation of Inner Mongolia Autonomous Region (\#2013MS1224), the Scientific Project of Affiliated Hospital of Inner Mongolia Medical University (\#NYFY2010YB006), the Youth Innovation Fund of Inner Mongolia Medical University (\#NY2010QN002), and the Key Scientific Fund of Affiliated Hospital of Inner Mongolia Medical University (\#NYFYZD20130158).

\section{REFERENCES}

Angheloiu GO, van de Poll SW, Georgakoudi I, Motz JT, et al. (2012). Intrinsic versus laser-induced fluorescence spectroscopy for coronary atherosclerosis: a generational comparison model for testing diagnostic accuracy. Appl. Spectrosc. 66: 1403-1410.

Balasegaram M, Ritmeijer K, Lima MA, Burza S, et al. (2012). Liposomal amphotericin B as a treatment for human leishmaniasis. Expert Opin. Emerg. Drugs 17: 493-510.

Butsch F, Faulde M, Debus A, Bogdan C, et al. (2012). Two cases of successful treatment of multilesional cutaneous leishmaniasis with liposomal amphotericin B. J. Dtsch. Dermatol. Ges. 11: 83-85.

Cacciapuoti F (2012). Lowering homocysteine levels with folic acid and B-vitamins do not reduce early atherosclerosis, but could interfere with cognitive decline and Alzheimer's disease. J. Thromb. Thrombolysis 36: 258-262.

Chen L, Ding L, Zhang H, Li J, et al. (2012). Dynamic microwave-assisted extraction coupled with on-line spectrophotometric determination of safflower yellow in Flos carthami. Anal. Chim. Acta 580: 75-82.

Hyafil F, Feldman L, Le Guludec D and Fayad ZA (2012). Evaluating efficacy of pharmaceutical interventions in atherosclerosis: role of magnetic resonance imaging and positron emission tomography. Mt. Sinai J. Med. 79: 689-704.

Jung SI, Shin JH, Choi HJ, Ju MY, et al. (2012). Antifungal susceptibility to amphotericin B, fluconazole, voriconazole, and flucytosine in Candida bloodstream isolates from 15 tertiary hospitals in Korea. Ann. Lab. Med. 32: 426-428.

Kurihara O, Takano M, Yamamoto M, Shirakabe A, et al. (2012). Impact of prediabetic status on coronary atherosclerosis: a multivessel angioscopic study. Diabetes Care 36: 729-733. 
Li Y, Zhang ZY and Zhang JL (2010). Determination of hydroxysafflor yellow A in rat plasma and tissues by highperformance liquid chromatography after oral administration of safflower extract or safflor yellow. Biomed. Chromatogr. 21: 326-334.

Liu B, Luo C, Ouyang L, Mu S, et al. (2011). An experimental study on the effect of safflower yellow on tendon injuryrepair in chickens. J. Surg. Res. 169: e175-e184.

Martinet W, De Meyer I, Verheye S, Schrijvers DM, et al. (2012). Drug-induced macrophage autophagy in atherosclerosis: for better or worse. Basic Res. Cardiol. 108: 321-327.

Mohamed-Ahmed AH, Brocchini S and Croft SL (2012). Recent advances in development of amphotericin B formulations for the treatment of visceral leishmaniasis. Curr. Opin. Infect. Dis. 25: 695-702.

Monteiro J, Li FJ, Maclennan M, Rabalski A, et al. (2011). Menhaden oil, but not safflower or soybean oil, aids in restoring the polyunsaturated fatty acid profile in the novel delta-6-desaturase null mouse. Lipids Health Dis. 11: 60-66.

Raz-Pasteur A, Gamliel-Lazarovich A, Coleman R and Keidar S (2012). Eplerenone reduced lesion size in early but not advanced atherosclerosis in apolipoprotein e-deficient mice. J. Cardiovasc. Pharmacol. 60: 508-512.

Wang CC, Choy CS, Liu YH, Cheah KP, et al. (2011). Protective effect of dried safflower petal aqueous extract and its main constituent, carthamus yellow, against lipopolysaccharide-induced inflammation in RAW264.7 macrophages. J. Sci. Food Agric. 91: 218-225.

Wei DH, Jia XY, Liu YH, Guo FX, et al. (2012). Cathepsin L stimulates autophagy and inhibits apoptosis of ox-LDLinduced endothelial cells: Potential role in atherosclerosis. Int. J. Mol. Med. 31: 400-406.

Xi SY, Zhang Q, Liu CY, Xie H, et al. (2012). Effects of hydroxy safflower yellow-A on tumor capillary angiogenesis in transplanted human gastric adenocarcinoma BGC-823 tumors in nude mice. J. Tradit. Chin. Med. 32: 243-248.

Zhao Z, Song G, Tian H, Yu Y, et al. (2012). Triacetyl-3-hydroxyphenyladenosine, a derivative of cordycepin, attenuates atherosclerosis in apolipoprotein E-knockout mice. Exp. Biol. Med. 237: 1262-1272. 